\title{
Synergistic inhibitory effects of an engineered antibody-like molecule ATF-Fc and trastuzumab on tumor growth and invasion in a human breast cancer xenograft mouse model
}

\author{
HONGWEI ZHOU ${ }^{1 *}$, HONGWEI WANG ${ }^{2 *}$, GUANGYUAN YU ${ }^{3 *}$, ZHIHONG WANG ${ }^{1}$, \\ $\mathrm{XI} \mathrm{ZHENG}^{1}$, HAIFENG DUAN ${ }^{4}$ and JUNZHONG SUN ${ }^{1}$ \\ Departments of ${ }^{1}$ Geriatric Oncology, ${ }^{2}$ Pathology and ${ }^{3}$ Medicine, The First Affiliated Hospital of Chinese PLA \\ General Hospital, Beijing 100048; ${ }^{4}$ Beijing Institute of Radiation Medicine, Beijing 100039, P.R. China
}

Received February 10, 2016; Accepted May 11, 2017

DOI: $10.3892 / \mathrm{ol} .2017 .6896$

\begin{abstract}
The overexpression of the oncogene human epidermal growth factor receptor 2 (HER-2) has been associated with decreased disease-free survival and is a marker of poor prognosis of invasive breast cancer. Although the high efficacy of trastuzumab, a drug that targets the HER-2 oncogene, has been widely recognized, the efficiency of the treatment remains at $\sim 30 \%$. Therefore, novel effective treatments are required for patients with recurrent metastatic breast cancer. The present study aimed to investigate the effects of an engineered antibody-like molecule administered alone or in combination with trastuzumab on the tumor growth and metastasis of HER-2-positive breast cancer. Another aim was to investigate novel cancer therapies for HER-2-positive breast cancer. The engineered antibody-like molecule consists of the amino-terminal fragment (ATF) of human urokinase-type plasminogen (uPA) and is conjugated with the $\mathrm{Fc}$ fragment of human immunoglobulin G1 (ATF-Fc). The anti-cancer effect of ATF-Fc (alone and in combination with trastuzumab) on tumor cells and in a nude mouse tumor model was evaluated
\end{abstract}

Correspondence to: Professor Junzhong Sun, Department of Geriatric Oncology, The First Affiliated Hospital of Chinese PLA General Hospital, 51 Fucheng Road, Beijing 100048, P.R. China E-mail: sjunzh07@163.com

Professor Haifeng Duan, Beijing Institute of Radiation Medicine, 27 Taiping Road, Beijing 100039, P.R. China

E-mail: duanhf0720@yahoo.com.cn

Abbreviations: HER-2, human epidermal growth factor receptor 2; uPA, urokinase-type plasminogen activator; CHO, Chinese hamster ovary; SPF, specific-pathogen-free; ELISA, enzyme-linked immunosorbent assay; IHC, immunohistochemistry; FISH, fluorescence in situ hybridization

*Contributed equally

Key words: urokinase, therapeutic antibody, human epidermal growth factor receptor 2, breast cancer, biological therapy by detecting the expression of uPA, urokinase plasminogen activator receptor (UPAR) and HER-2. In vitro experiments demonstrated that specifically blocking the UPA-uPAR and HER-2 signaling pathways may effectively promote the apoptosis of breast cancer cells. Additionally, ATF-Fc-induced cell death in HER-2-positive breast cancer cells was observed in vivo. When ATF-Fc was administered in combination with trastuzumab, cell death was increased and breast cancer metastasis was reduced. The novel engineered antibody-like molecule ATF-Fc was able to inhibit HER-2-positive breast cancer cell growth and metastasis by interfering with uPA and its receptor (UPA-uPAR) system. Additionally, the antibody-like molecule exhibits a synergistic inhibitory effect when administered in combination with trastuzumab.

\section{Introduction}

Novel effective treatments are required for patients with recurrent metastatic breast cancer, particularly for those who fail to respond to third-line and subsequent lines of chemotherapy (1). Overexpression of the oncogene human epidermal growth factor receptor 2 (HER-2) is an independent indicator of poor prognosis (2). HER-2 overexpression has been associated with decreased survival time and resistance to certain chemoand endocrine therapies in patients with breast cancer $(3,4)$. Although the high efficacy of trastuzumab, a drug that targets the HER-2 oncogene, has been widely recognized, its efficiency remains at $\sim 30 \%(5,6)$. Patients who fail to response to third-line or subsequent lines of chemotherapy are usually of poor physical condition and have difficulty tolerating additional chemotherapy (7). Therefore, methods for optimizing effective biological therapies to improve patient quality of life and survival times are urgently required in clinical practice.

Tumor initiation and development are a result of multiple genetic abnormalities; therefore, blocking the expression of cancer-promoting genes at multiple points and along numerous signaling pathways may theoretically limit tumor development (8). Studies have demonstrated that the expression of the urokinase-type plasminogen activator (uPA) and its receptor (UPAR) system is significantly increased in various human tumor tissues, including breast, lung and colorectal 
cancer (9-11). Furthermore, uPA-uPAR expression has been significantly associated with tumor growth and patient prognosis (12-14). As uPA-uPAR has key roles in tumor metastasis and growth, it has become an ideal candidate for targeted cancer therapy. Interference with UPA-uPAR interactions not only inhibits tumor infiltrations but also is able to block tumor angiogenesis, thereby effectively controlling tumor growth $(15,16)$. In a previous study by the authors, an antibody-like molecule comprised of the amino-terminal fragment (ATF) of UPA and is conjugated to the Fc fragment of immunoglobulin (Ig) G1 (ATF-Fc), was engineered. The specific inhibitory effect of the antibody-like molecule on tumors was evaluated (17).

In the present study, the authors proposed using the ATF-Fc fusion protein to target uPA-uPAR and trastuzumab signaling pathways, and to inhibit HER-2 oncogene function in breast cancer. In addition, the effect of ATF-Fc in combination with trastuzumab on the tumor apoptosis and metastasis of HER-2-overexpressing breast cancer was evaluated in vivo.

\section{Materials and methods}

Preparation of an engineered antibody-like molecule ATF-FC. ATF-Fc-2E9, a recombinant Chinese hamster ovary cell line which was stably transfected with ATF-Fc (17), was gifted by Professor Haifeng Duan (Beijing Institute of Radiation Medicine, Beijing, China). The ATF-Fc expression level of the ATF-Fc-2E9 cell line was $\sim 20 \mu \mathrm{g} / 10^{6}$ cells/day. A fusion protein with a molecular weight of $\sim 96 \mathrm{kDa}$ under non-reducing conditions and $\sim 45 \mathrm{kDa}$ under reducing conditions was obtained, according to the protocols previously published $(17,18)$. The fusion protein consists of the ATF of human UPA and is conjugated with the Fc fragment of human IgG1.

Experimental drug trastuzumab. The monoclonal antibody trastuzumab (catalog no., 10172501; $440 \mathrm{mg} / \mathrm{vial}$ ) was provided by (Roche Diagnostics, Basel, Switzerland), and was freshly prepared prior to each experiment. Briefly, each $440 \mathrm{mg}$ vial of trastuzumab was reconstituted with $20 \mathrm{ml}$ of bacteriostatic water for injection, containing $1.1 \%$ benzyl alcohol as a preservative to yield a multi-dose solution containing $21 \mathrm{mg} / \mathrm{ml}$ trastuzumab. The drug was then diluted to the required concentration $(0.12 \mathrm{mg} / \mathrm{ml})$ using sterile saline.

Cell lines and experimental animals. The EC-109 human esophageal cancer cell line and the MCF-7 and SK-BR-3 human breast cancer cell lines were purchased from the Cell Culture Center of the Chinese Academy of Science (Beijing, China). The EC-109 and MCF-7 cells were maintained in Dulbecco's modified Eagle's medium (HyClone; GE Healthcare Life Sciences, Logan, UT, USA), while the SK-BR-3 cells were maintained in RPMI-1640 (HyClone; GE Healthcare Life Sciences), supplemented with $10 \%$ fetal bovine serum (Gibco; Thermo Fisher Scientific, Inc., Waltham, MA, USA), 100 U/ml streptomycin and $100 \mathrm{U} / \mathrm{ml}$ penicillin (Beijing Solarbio Science \& Technology Co., Ltd., Beijing, China). All the cells were incubated in a humidified incubator containing $5 \% \mathrm{CO}_{2}$ at $37^{\circ} \mathrm{C}$. The experimental models used were 35 4-6-week-old female BALB/C nude mice, which were purchased from the Animal Center of the Peking Union Medical College Hospital
(Beijing, China). The experimental protocol was approved by the Experimental Animal Center of China. All experiments were performed according to the standards of animal care as outlined in the Guide for the Care and Use of Experimental Animals of Peking Union Medical College. Briefly, the mice were maintained in an air-conditioned specific-pathogen-free laboratory with $12 / 12$-h light-dark cycle and $24 \pm 1^{\circ} \mathrm{C}$ and $5 \pm 5 \%$ relative humidity, and had free access to food and water during the study. Following one week of acclimatization, the animals were subcutaneously injected into the right flank with SK-BR-3 cells suspended in serum-free medium in the exponential growth stage at a dosage of $1 \times 10^{7}$ cells/nude mouse. The period of tumor formation was 7-10 days. When the tumors reached a volume of $\sim 3 \mathrm{~cm}^{3}$ calculated as previously described $(19,20)$, the mice were sacrificed and the tumor tissues were dissected, minced, ground, homogenized and re-injected into another four groups of the same type of mice ( 7 mice per group). Following 1-2 weeks, tumors developed, and the animals were randomly grouped for subsequent experiments.

Detection of $u P A$ and $U P A R$ expression in tumor cell lines. The serum-free supernatant from the EC-109, MCF-7 and SK-BR-3 cell cultures was collected and centrifuged at $850 \mathrm{x} \mathrm{g}$ for 10 min at $4^{\circ} \mathrm{C}$ (ThermoHeraeus Multifuge X1R; Thermo Fisher Scientific, Inc.) for detection of uPA (three samples from each cell line). Triple wells were used in each cell line. An ELISA was performed to determine the level of uPA secreted from the cells using an uPA ELISA kit (catalog no., ab108917; Abcam, Cambridge, MA, USA) The assay was performed according to the manufacturer's instructions. The extracellular level of uPAR was determined using a FACscan flow cytometer (BD Biosciences, San Jose, CA, USA) (3 samples in each cell line). The procedure is briefly described, as follows: $1 \times 10^{6} \mathrm{EC}-109$, MCF-7 and SK-BR-3 cells were incubated with an anti-uPAR antibody (10 $\mu \mathrm{g}$ per $1 \times 10^{6}$ cells) (catalog no., sc-13522; Santa Cruz Biotechnologies, Dallas, USA) for $2 \mathrm{~h}$ at $4^{\circ} \mathrm{C}$. The cells were washed three times with PBS containing $0.05 \%$ Tween-20 to remove the unbound antibody and then incubated with a fluorescein isothiocyanate-conjugated secondary antibody (dilution, 1:500; catalog no., ab6785; Abcam) for $30 \mathrm{~min}$ at room temperature. The normal mouse IgG (cat. no., sc-2025; Santa Cruz) were used as the controls. The flow cytometry results were analyzed using a FACScan instrument (CellQuest Pro Software 5.1; BD Biosciences).

HER-2 oncogene immunohistochemistry (IHC) and fluorescence in situ hybridization (FISH). Anti-HER-2 monoclonal antibody (cat. no., ZM0065; dilution, 1:200) and PV6000 detection kit (both Zhongshan Golden Bridge Biotechnology Co., Ltd., Beijing, China) were used for IHC analysis. The IHC detection of HER-2 protein was performed according to the manufacturer's instructions. Plasmalemma exhibiting brown particles was considered positively stained. Based on the intensity and continuity of the color, the staining results were classified as 'negative' for no color (0), 'positive' for light-yellow (1+), 'strong positive' for brownish-yellow (2+) and 'extreme positive' for brown (3+).

The HER-2 gene FISH detection kit was provided by Daan Gene Co., Ltd. FISH detection of HER-2 was performed according to the manufacturer's instructions. In a clear tumor 
area, the total number of HER-2 (red) and CEP-17 (green) signals in 60 cell nuclei were counted, and the ratio of the number of HER-2 to CEP-17 signals was calculated. The following assessment criteria were used: If the ratio was $\geq 2.2$, the result was considered positive (with amplification); if the ratio was $<1.8$, the result was considered negative (without amplification); if the ratio was close to borderline values (1.8-2.2), the signals in an additional 20 nuclei were counted (21).

In vitro analysis of apoptosis status in a breast cancer cell line. SK-BR-3 cells in the exponential growth stage were seeded in 96-well tissue culture plates (Costar; Corning Incorporated, Corning, NY, USA) at a density of $5 \times 10^{4}$ cells/well. When the cells reached $65-70 \%$ confluency, ATF-Fc (final concentration of $50 \mu \mathrm{g} / \mathrm{ml}$ ), trastuzumab (final concentration of $10 \mathrm{mg} / \mathrm{ml}$ ) or ATF-Fc $(50 \mu \mathrm{g} / \mathrm{ml})$ plus trastuzumab $(10 \mathrm{mg} / \mathrm{ml})$ was added. Saline was added to the control group. The cells were continuously cultured for an additional $72 \mathrm{~h}$ at $37^{\circ} \mathrm{C}$ in a humidified incubator containing $5 \% \mathrm{CO}_{2}$. The culture medium was subsequently removed and the cells were collected. The cells were then stained with Annexin-V and 7-amino-actinomycin D (7-AAD), according to the manufacturer's instructions (BD Biosciences). Briefly, subsequent to washing with ice-cold PBS, SK-BR-3 cells were resuspended in $200 \mu \mathrm{l}$ of $1 \mathrm{X}$ annexin-V binding buffer. Next, $5 \mu \mathrm{l}$ of annexin-V-APC and $5 \mu \mathrm{l}$ of 7 -AAD were added to the tubes and incubated for $10 \mathrm{~min}$ at $4^{\circ} \mathrm{C}$ in the dark. The samples were placed on ice and the cellular apoptotic rate was determined using flow cytometry. Triplicate wells were used in the experimental and in the control groups.

In vivo analysis of tumor growth and metastasis in breast cancer xenograft mouse model. As aforementioned, female BALB/C nude mice inoculated with SK-BR-3 cells were randomly grouped for experimentation when the size of the tumors reached $100 \mathrm{~mm}^{3}$. Each group consisted of seven mice. The following four experimental groups were included: ATF-Fc alone; trastuzumab alone; ATF-Fc plus trastuzumab; a blank control group. ATF-Fc (10 mg/kg) was administered once every two days via tail vein injection for three continuous weeks, and trastuzumab $(6 \mathrm{mg} / \mathrm{kg})$ was administered once a week for two weeks. The medication regimen of ATF-Fc plus trastuzumab group was ATF-Fc $(10 \mathrm{mg} / \mathrm{kg})$ administered once every 2 days via tail vein injection for 3 continuous weeks, combined with trastuzumab $(6 \mathrm{mg} / \mathrm{kg})$ administered once a week for 2 weeks. An equal volume of saline ( $1 \mathrm{ml} /$ time) was used once every two days for the control group. Following drug administration, the mice were observed for 3 weeks. The longitudinal and transverse diameters of the tumors were measured every other day, and the tumor volume was calculated as previously described $(19,20)$. After 3 weeks, the animals were sacrificed. The tumor tissues were isolated and weighed to calculate the tumor inhibition rate. The mice were dissected to examine the liver metastasis status. The number of mice with liver metastasis and the number of metastatic foci in each liver were recorded. Briefly, specimens for histological examination were fixed in $10 \%$ formalin for $24 \mathrm{~h}$. To ensure systematic uniform and random sampling, the entire liver of each animal was cut transversally to the portal vein into $2 \mathrm{~mm}$

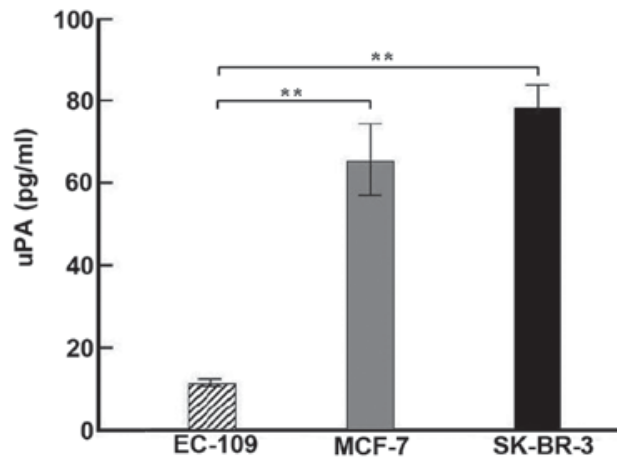

Figure 1. Extracellular level of uPA in EC-109, MCF-7 and SK-BR-3 cells The extracellular level of uPA in EC-109, MCF-7 and SK-BR-3 cells was determined via ELISA. Each experiment was repeated three times. The data are presented as the mean \pm standard deviation. The uPA expression levels in the three types of cells were $12.32 \pm 1.18,63.77 \pm 9.26$ and $78.62 \pm 6.09 \mathrm{pg} / \mathrm{ml}$, respectively. The SK-BR-3 and MCF-7 cells exhibited significantly increased levels of uPA expression compared with EC-109 cells $\left({ }^{* *} \mathrm{P}<0.01\right)$. uPA, urokinase-type plasminogen activator.

thick parallel sections, the cut surfaces were examined and the specimens were embedded in paraffin. Sections $(5 \mu \mathrm{m})$ were cut and stained with hematoxylin and eosin and Periodic Acid-Schiff (G1281; Beijing Solarbio Science \& Technology Co., Ltd.) to confirm the presence of cancerous cells by light microscopy. The equation for calculating tumor volume is as follows $(19,20)$ :

\section{$\mathrm{V}=\mathrm{LxD}^{2} / 2$}

( $\mathrm{L}$, maximum diameter of the tumor; $\mathrm{D}$, minimum diameter of the tumor). The equation for calculating the tumor inhibition rate of drug effect based on the tumor weight is as follows:

$$
\text { (a-b)/ax100\% }
$$

(a, mean tumor weight in the control group; b, mean tumor weight in the treatment group).

Statistical analysis. The results are presented as the mean \pm standard deviation. Group differences were analyzed with the non-parametric Kruskal-Wallis test. Comparisons of the tumor weight and the liver metastasis rate in the nude mice with tumors were analyzed using the Pearson $\chi^{2}$ test. In experiments involving histology or flow cytometry, the figures presented are representative of $\geq 3$ experiments performed on different days on the tissue sections or tumor cells. All statistical assessments were two-sided, and $\mathrm{P}<0.05$ was considered to indicate a statistically significant difference. All analyses were performed using SPSS software (version 15.0; SPSS Inc., Chicago, IL, USA).

\section{Results}

Determination of $U P A, U P A R$ and HER-2 expression in tumors cell lines. The expression levels of UPA, UPAR, and HER-2 in EC-109, MCF-7 and SK-BR-3 cells were determined as aforementioned. As presented in Fig. 1, the expression level of uPA in EC-109, MCF-7 and SK-BR-3 cells was 12.32 $\pm 1.18,63.77 \pm 9.26$, and $78.62 \pm 6.09 \mathrm{pg} / \mathrm{ml}$, respectively. The uPAR expression 


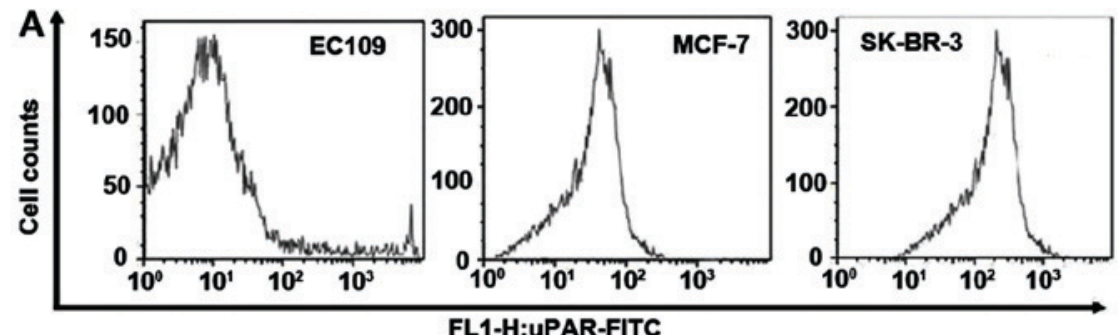

B

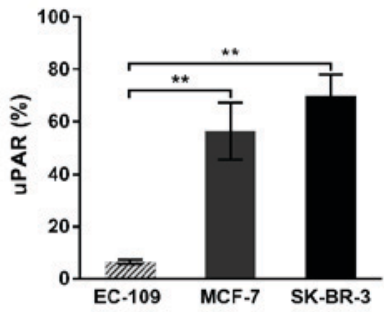

C

EC-109

MCF-7

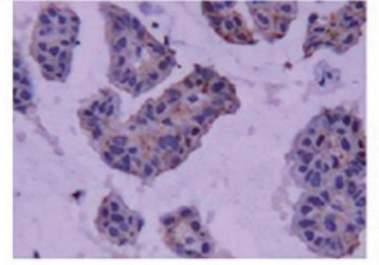

D

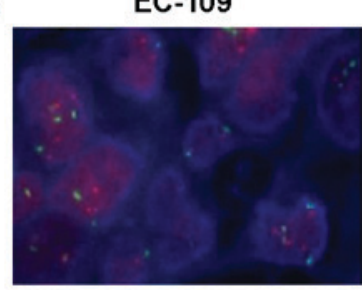

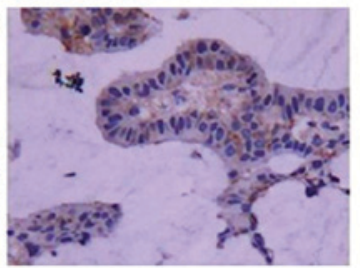

MCF-7

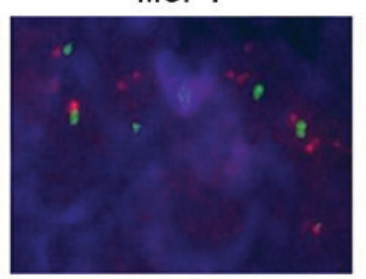

SK-BR-3

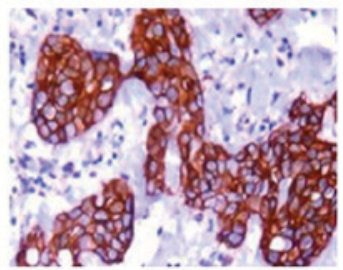

SK-BR-3

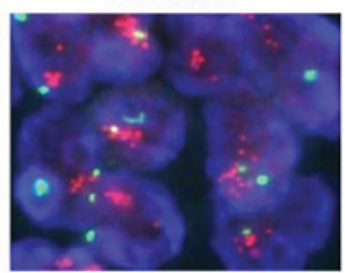

Figure 2. Expression of uPAR and HER-2 in various cell lines. (A) The uPAR expression levels in EC-109, MCF-7 and SK-BR-3 cells were determined by flow cytometry. The uPAR expression rate was $6.5,56.32$ and $69.87 \%$. (B) The SK-BR-3 cells exhibited significantly increased levels of expression compared with EC-109 cells ( $\left.{ }^{* *} \mathrm{P}<0.01\right)$. (C) The HER-2 protein expression was determined via immunohistochemistry in EC-109, MCF-7 and SK-BR-3 cells. HER-2 was not present in EC-109 and MCF-7 cells. By contrast, the SK-BR-3 cells exhibited brown staining, indicating positive expression. (D) The HER-2 gene expression was detected by fluorescence in situ hybridization. The SK-BR-3 cells exhibited positive amplification with a HER-2/CEP-17 ratio of 4.38, whereas the EC-109 and MCF-7 cells were negative for amplification and had HER-2/CEP-17 ratios of 1.41 and 1.26, respectively. FITC, fluorescein isothiocyanate; HER-2, human epidermal growth factor receptor; uPAR, urokinase plasminogen activator receptor; uPA, urokinase-type plasminogen activator.

rate was 6.5, 56.32 and $69.87 \%$, respectively (Fig. 2A and B). The SK-BR-3 cells expressed significantly increased levels of UPA and UPAR compared with EC-109 cells, ( ${ }^{* *} \mathrm{P}$-value<0.01). The HER-2 expression, as determined by IHC, revealed that EC-109 and MCF-7 cells were negative for HER-2 expression and that the nuclei were blue. By contrast, the SK-BR-3 cells exhibited strong positive expression, and the nuclear membrane appeared dark-brown (Fig. 2C). FISH analysis verified that the HER-2 gene was amplified in the SK-BR-3 cells (Fig. 2D). These results suggested that SK-BR-3 cells exhibit elevated levels of UPA and UPAR expression, and high levels of HER-2 expression. Therefore, SK-BR-3 cells were selected for further experiments to determine the inhibitory effect of ATF-Fc in combination with trastuzumab on cells.

Apoptosis status of breast cancer cells following treatment with ATF-Fc or trastuzumab alone and in combination. Following the treatment of SK-BR-3 cells (in the exponential growth stage) with ATF-Fc $(50 \mu \mathrm{g} / \mathrm{ml})$ or trastuzumab $(10 \mathrm{mg} / \mathrm{ml})$ alone and in combination for $72 \mathrm{~h}$, the cells were stained with Annexin-V and 7-AAD, and then analyzed by flow cytometry. Representative dot plots indicating the presence of early and late apoptotic tumor cells are depicted in Fig. 3A. As illustrated in Fig. 3B, treatment with ATF-Fc or trastuzumab alone resulted in a cellular apoptotic rate of $42 \pm 7.79$ and $38 \pm 4.55 \%$, respectively. By contrast, treatment with a combination of ATF-Fc and trastuzumab resulted in an apoptotic rate of $83 \pm 6.58 \%$. The apoptotic rates of tumor cells were significantly higher in the ATF-Fc and trastuzumab combination treatment group, as compared with in the ATF-Fc $(\mathrm{P}<0.01)$ or trastuzumab alone groups $(\mathrm{P}<0.01)$. These results indicate that specifically blocking the uPA-uPAR and HER-2 pathways may effectively promote the apoptosis of breast cancer cells in vitro.

In vivo analysis of inhibition of tumor cell proliferation. Tumor-bearing nude mice were administered ATF-Fc alone, trastuzumab alone or a combination of ATF-Fc and trastuzumab according to the protocols previously described. The control group received saline via tail vein injection. In the 

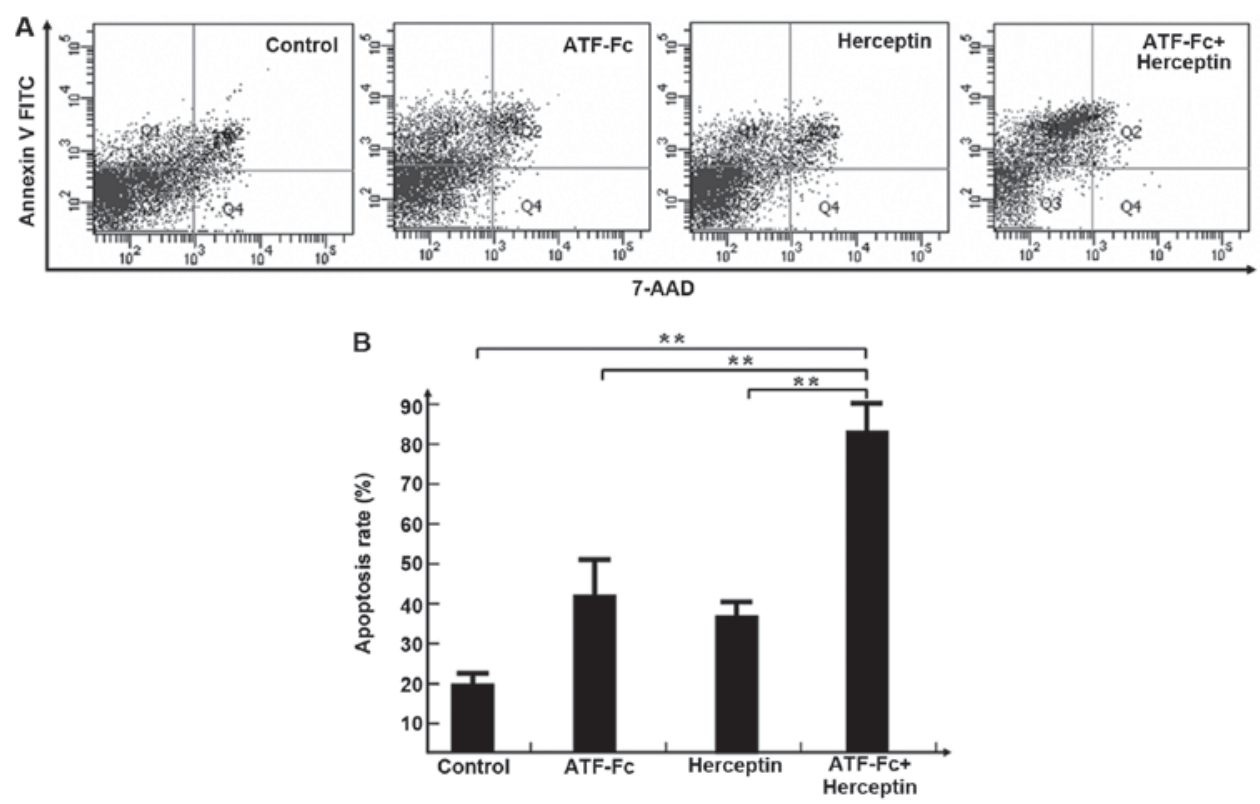

Figure 3. Effect of ATF-Fc and trastuzumab on apoptosis of SK-BR-3 cells. Apoptosis was analyzed using Annexin-V in the SK-BR-3 cells following treatment for $72 \mathrm{~h}$. Flow cytometry was used to determine the percentage of cells positive for annexin-V and/or 7-AAD. (A) Representative dot plots of three independent analyses. Early apoptotic cells were identified as Annexin-V positive and 7-AAD negative. Late apoptotic cells were identified as positive for Annexin $\mathrm{V}$ and 7-AAD staining. (B) The apoptotic rate of tumor cells was significantly increased in the ATF-Fc $(50 \mu \mathrm{g} / \mathrm{ml})$ plus trastuzumab (10 $\mathrm{mg} / \mathrm{ml})$ treatment group, as compared with in the ATF-Fc $(50 \mu \mathrm{g} / \mathrm{ml})$ or trastuzumab $(10 \mathrm{mg} / \mathrm{ml})$ alone groups ( $\left.{ }^{* *} \mathrm{P}<0.01\right)$. 7-AAD, 7-amino-actinomycin D; ATF-Fc, amino-terminal fragment of uPA-Fc fragment of human immunoglobulin G1; FITC, fluorescein isothiocyanate.
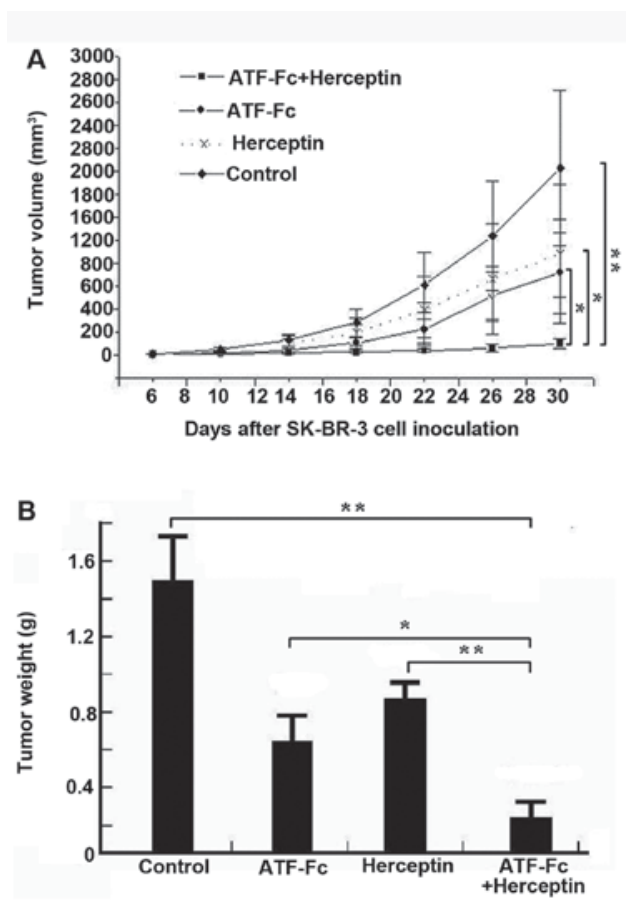

Figure 4. In vivo analysis of the inhibitory effect of ATF-Fc, trastuzumab and ATF-Fc plus trastuzumab on SK-BR-3 cell proliferation. A SK-BR-3 human breast cancer xenograft BALB/C (neu) nude mouse model was established. (A) The curve represents the change in tumor volume following treatment with ATF-Fc, trastuzumab or ATF-Fc plus trastuzumab. The ATF-Fc $(10 \mathrm{mg} / \mathrm{kg})$ in combination with trastuzumab $(6 \mathrm{mg} / \mathrm{kg})$ significantly inhibited SK-BR-3 cell growth. After 30 days, the tumor volume in the group treated with ATF-Fc plus trastuzumab was significantly decreased, as compared with in the control group and in the groups treated with ATF-Fc or trastuzumab alone ( $\mathrm{P}<0.05$ and $\left.{ }^{* *} \mathrm{P}<0.01\right)(\mathrm{B})$ Change in tumor weight following treatment of the nude mice with ATF-Fc, trastuzumab or ATF-Fc plus trastuzumab. Treatment with ATF-Fc plus trastuzumab significantly reduced the SK-BR-3 tumor volume compared with ATF-Fc alone, trastuzumab alone and the control. ATF-Fc, amino-terminal fragment of uPA -Fc fragment of human immunoglobulin G1. present study, the mice did not exhibit symptoms of fever, vomiting, diarrhea or skin rash following administration of ATF-Fc or trastuzumab alone or combination with ATF-Fc and trastuzumab. As illustrated in Fig. 4A, compared with the control group, treatment with ATF-Fc in combination with trastuzumab significantly reduced SK-BR-3 tumor volume $(\mathrm{P}<0.01)$. The inhibitory effect of the combination treatment was significantly higher, as compared with treatment with either ATF-Fc alone $(\mathrm{P}<0.05)$ or trastuzumab alone $(\mathrm{P}<0.05)$. Fig. 4B indicates that the treatment of ATF-Fc in combination with trastuzumab significantly reduced the SK-BR-3 tumor volume compared with the control group, ATF-Fc alone and trastuzumab alone $(\mathrm{P}<0.05$ and $\mathrm{P}<0.01$, respectively). The tumor inhibition rates were 60,74 , and $84 \%$ for ATF-Fc alone, trastuzumab alone and combination treatments, respectively.

Inhibitory effect on liver metastasis in tumor-bearing nude mice. Following the administration of the drugs to the mice for three weeks, the mice were sacrificed and dissected. The tissues were subsequently evaluated for liver metastasis status, and the number of metastatic foci was determined. Compared with the control group, treatment with a combination of ATF-Fc and trastuzumab had a significant inhibitory effect on liver metastasis in the tumor-bearing nude mice. The differences in the rate of liver metastasis and the number of metastatic foci between the group treated with trastuzumab or ATF-Fc or ATF-Fc plus trastuzumab and the control group were statistically significant. In addition, the administration of ATF-Fc in combination with trastuzumab significantly reduced the rate of liver metastasis and the number of metastatic foci, compared with ATF-Fc or trastuzumab alone (Table I). These results suggest that treatment with ATF-Fc in combination 
Table I. Inhibitory effect of trastuzumab, ATF-Fc or ATF-Fc plus trastuzumab on liver metastasis.

\begin{tabular}{|c|c|c|}
\hline Group & $\begin{array}{l}\text { Metastasis } \\
\text { rate }(\%)\end{array}$ & $\begin{array}{c}\text { Number } \\
\text { of foci }\end{array}$ \\
\hline Control & $71.4(5 / 7)$ & $69.8 \pm 11.9$ \\
\hline${ }^{a}$ Trastuzumab (6 mg/kg) & $42.9(3 / 7)$ & $52.5 \pm 9.6$ \\
\hline aATF-Fc (10 mg/kg) & $28.6(2 / 7)$ & $44.8 \pm 5.1$ \\
\hline${ }^{a-c}$ ATF-Fc plus trastuzumab & $14.3(1 / 7)$ & $13.7 \pm 4.8$ \\
\hline
\end{tabular}

${ }^{\mathrm{a}} \mathrm{P}<0.05$ vs. control; ${ }^{\mathrm{b}} \mathrm{P}<0.01$ vs. trastuzumab; ${ }^{\mathrm{c}} \mathrm{P}<0.01$ vs. ATF-Fc. Results are presented as the mean \pm standard deviation. ATF-Fc, amino-terminal fragment of uPA-Fc fragment of human immunoglobulin G1.

with trastuzumab may increase the inhibitory effect on breast cancer metastasis.

\section{Discussion}

Chemotherapy is an effective method for treating malignant tumors. However, due to the presence of primary and secondary drug resistance, tumors in numerous patients ultimately progress and their health deteriorates (22). Therefore, studies in oncology are constantly investigating and searching for more effective therapeutics.

Targeted (biological) therapy has an increasingly critical role in treating malignant tumors. For example, rituximab specifically targets cluster of differentiation (CD)20 (23). Trastuzumab specifically targets HER-2 (24), and bevacizumab specifically targets vascular endothelial growth factor $(25,26)$. Rituximab, trastuzumab and bevacizumab have already exhibited notable efficacy in the treatment of multiple human malignant tumors (27).

The uPA-uPAR system has an essential role in tumor growth and metastasis, and overexpression of these molecules is strongly associated with poor prognosis in a variety of malignant tumors $(12-13,28)$. The monoclonal antibody against uPA or UPAR has been demonstrated to be effective in inhibiting the proliferation, migration and invasiveness of cancer in vitro $(29,30)$. Another known antagonist of uPA-uPAR is the ATF of higher-molecular-weight uPA, which contains an epidermal growth factor-like domain and a kringle domain (31). ATF is a fragment of uPA without enzyme activity that exhibits a strong affinity for uPAR (32). In 1993, Crowley et al (33) constructed an antibody-like molecule that is comprised of 1-137 amino acids (AA) of uPA and is conjugated with the $\mathrm{Fc}$ fragment of an IgG molecule. The binding target of this molecule was UPAR, and the molecule exhibited an inhibitory effect on tumor cell dissemination (33). However, the anti-tumor effect of this molecule is not ideal. Further study revealed that the uPA fragment in Crowley's construct contained the enzymatic site for plasmin (uPA, AA135 and 136). This defect meant that the molecule is susceptible to enzymatic digestion in tumor tissues that are rich in plasmin, thus preventing it from targeting UPAR (17).

In order to overcome this defect in the present study, a novel ATF-Fc fragment that did not contain the enzymatic site for plasmin was constructed. In vitro and in vivo studies demonstrated that ATF-Fc exhibits an inhibitory effect on tumor growth, metastasis and angiogenesis. These findings indicate that ATF-Fc is a promising antibody drug with the potential to serve a crucial role in anti-tumor therapy in the future (17).

Overexpression of HER-2 or UPAR in breast cancer has been associated with an increased aggressive primary tumor phenotype and a poor prognosis $(4,34)$. Although the role of the HER-2 oncogene has been recognized, $\leq 70 \%$ of HER-2-positive breast cancer patients exhibit primary resistance to trastuzumab (35). Additionally, $70 \%$ of the patients who effectively respond to the treatment eventually develop drug resistance-associated relapse within one year $(36,37)$. A number of studies have demonstrated that multiple genetic abnormalities are involved in tumor initiation, development and metastasis $(38,39)$. Therefore, ideal anti-tumors effects are unlikely to be achieved by blocking or inhibiting the expression of a single oncogene. A previous study suggested that the overexpression and gene amplification of HER-2 and UPAR occurred most frequently in the same individual tumor cells in primary breast carcinoma, and in the circulating tumor cells of patients with advanced breast carcinoma (40). Pierga et al (41) reported comparable results in disseminated tumor cells from the bone marrow of patients with breast cancer. These findings indicate that targeting HER-2 and uPAR together may provide a more efficient therapy for patients with breast cancer.

A previous study by the authors (17) demonstrated that treatment with ATF-Fc resulted in significant suppression of the growth and metastasis of xenograft human tumors (MCF-7 breast cancer and BGC-823 gastric cancer) in athymic nude mice. ATF-Fc could specifically inhibit the uPA-uPAR interaction, but trastuzumab did not have this effect. The present study indicates that when combined with trastuzumab, a drug used to target HER-2, ATF-Fc can synergistically inhibit HER-2-positive breast cancer cell proliferation and metastasis by interfering with the uPA-uPAR system. To identify an effective biological therapeutic method, the SK-BR-3 breast cancer cell line was selected, which overexpresses uPA/uPAR and HER-2, to establish a tumor-bearing animal model. Subsequently, the effect of ATF-Fc in combination with trastuzumab on the growth and metastasis of the tumors was determined by interfering with two pathways.

In the present study, significant inhibition of breast cancer cell proliferation has been demonstrated in vitro, and significant inhibition of tumor metastasis has been observed in vivo. The inhibitory effect of the combination treatment with trastuzumab and ATF-Fc was significantly increased compared with treatment with trastuzumab or ATF-Fc alone. However, the mechanisms of action of the combination treatment remain unknown.

Several studies (42-44) have suggested that epidermal growth factor receptor (EGFR) may function as a transducer of the signaling between uPAR to ERK, which indicates the existence of crosstalk between EGFR and uPAR signaling pathways. By contrast, it has been demonstrated that depletion of HER-2 and uPAR by small interfering RNA is able to suppress mitogen-activated protein kinase signaling pathways, resulting in a decrease of extracellular-signal-regulated kinase (ERK) activity and a high p38/ERK activity ratio, which is 
involved in the synergistic suppression of breast cancer cell growth (45). Therefore, crosstalk between HER-2 and UPAR signaling pathways may exist, and may be a potential underlying mechanism of the synergistic anti-tumor activity profiles of combined ATF-Fc and trastuzumab treatments in breast cancer cells. Together, these findings suggest that ATF-Fc has high therapeutic value for use in combination with trastuzumab in patients with HER-2-positive breast cancer, where primary resistance to trastuzumab is also present.

One limitation of the current study was that only one cell line, which overexpresses HER-2, was used to investigate the synergistic inhibitory effects of ATF-Fc and trastuzumab on tumor growth and invasion. Additionally, the potential underlying mechanism of the synergistic inhibitory effects has yet to be determined. These critical issues must be addressed in future investigations.

In summary, the present study demonstrates that a combination of ATF-Fc (an antibody-like molecule that targets uPAR) and trastuzumab, (a monoclonal antibody that targets HER-2) exhibits synergistic inhibitory effects on tumor growth and metastasis, and may serve a key role in the treatment of cancer in the future.

\section{Acknowledgements}

The present study was supported by the National Natural Science Foundation of China (grant nos. 81071839 and 30971297).

\section{References}

1. Saji S: Evolving approaches to metastatic breast cancer patients pre-treated with anthracycline and taxane. BioDrugs 27: 469-478, 2013.

2. Riou G, Mathieu MC, Barrois M, Le Bihan ML, Ahomadegbe JC, Bénard J and Lê MG: c-erbB-2 (HER-2/neu) gene amplification is a better indicator of poor prognosis than protein over-expression in operable breast-cancer patients. Int J Cancer 95: 266-270, 2001.

3. Pegram MD, Finn RS, Arzoo K, Beryt M, Pietras RJ and Slamon DJ: The effect of HER-2/neu overexpression on chemotherapeutic drug sensitivity in human breast and ovarian cancer cells. Oncogene 15: 537-547, 1997.

4. Wang GS, Zhu $\mathrm{H}$ and Bi SJ: Pathological features and prognosis of different molecular subtypes of breast cancer. Mol Med Report 6: 779-782, 2012.

5. Heyerdahl H, Abbas N, Brevik EM, Mollatt C and Dahle J: Fractionated therapy of HER2-expressing breast and ovarian cancer xenografts in mice with targeted alpha emitting 227Th-DOTA-p-benzyl-trastuzumab. PLoS One 7: e42345, 2012.

6. $\mathrm{Vu} \mathrm{T}$ and Claret FX: Trastuzumab: Updated mechanisms of action and resistance in breast cancer. Front Oncol 2: 62, 2012.

7. Maeda S, Saimura M, Minami S, Kurashita K, Nishimura R, Kai Y, Yano H, Mashino K, Mitsuyama S, Shimokawa M, et al: Efficacy and safety of eribulin as first-to third-line treatment in patients with advanced or metastatic breast cancer previously treated with anthracyclines and taxanes. Brest 32 : 66-72, 2017.

8. Berger C, Madshus IH and Stang E: Cetuximab in combination with anti-human IgG antibodies efficiently down-regulates the EGF receptor by macropinocytosis. Exp Cell Res 318: 2578-2591, 2012.

9. Meng S, Tripathy D, Shete S, Ashfaq R, Saboorian H, Haley B, Frenkel E, Euhus D, Leitch M, Osborne C, et al: uPAR and HER-2 gene status in individual breast cancer cells from blood and tissues. Proc Natl Acad Sci USA 103: 17361-17365, 2006.

10. Su CY, Liu YP, Yang CJ, Lin YF, Chiou J, Chi LH, Lee JJ, Wu AT, Lu PJ, Huang MS and Hsiao M: Plasminogen activator inhibitor-2 plays a leading prognostic role among protease families in non-small cell lung cancer. PLoS One 10: e0133411, 2015.
11. Märkl B, Renk I, Oruzio DV, Jähnig H, Schenkirsch G, Schöler C, Ehret W, Arnholdt HM, Anthuber M and Spatz H: Tumour budding, uPA and PAI-1 are associated with aggressive behaviour in colon cancer. J Surg Oncol 102: 235-241, 2010.

12. Kim TD, Song KS, Li G, Choi H, Park HD, Lim K, Hwang BD and Yoon WH: Activity and expression of urokinase-type plasminogen activator and matrix metalloproteinases in human colorectal cancer. BMC Cancer 6: 211, 2006.

13. Foekens JA, Peters HA, Look MP, Portengen H, Schmitt M, Kramer MD, Brünner N, Jänicke F, Meijer-van Gelder ME, Henzen-Logmans SC, et al: The urokinase system of plasminogen activation and prognosis in 2780 breast cancer patients. Cancer Res 60: 636-643, 2000.

14. Look MP, van Putten WL, Duffy MJ, Harbeck N, Christensen IJ, Thomssen C, Kates R, Spyratos F, Fernö M, Eppenberger-Castori S, et al: Pool analysis of prognostic impact of urokinase-type plasminogen activator and its inhibitor PAI-1 in 8377 breast cancer patients. J Natl Cancer Inst 94: 116-128, 2002.

15. Laufs S, Schumacher J and Allgayer H: Urokinase-receptor (u-PAR): An essential player in multiple games of cancer: A review on its role in tumors progression, invasion, metastasis, proliferation/dormancy, clinical outcome and minimal residual disease. Cell Cycle 5: 1760-1771, 2006.

16. Sidenius $\mathrm{N}$ and Blasi F: The urokinase plasminogen activator system in cancer: Recent advances and implication for prognosis and therapy. Cancer Metastasis Rev 22: 205-222, 2003.

17. Hu XW, Duan HF, Gao LH, Pan SY, Li YM, Xi Y, Zhao SR, Yin L, Li JF, Chen HP and Wu CT: Inhibition of tumors growth and metastasis by ATF-Fc, an engineered antibody targeting urokinase receptor. Cancer Biol Ther 7: 651-659, 2008.

18. Hu X, Xiao C, Huang Z, Guo Z, Zhang Z and Li Z: Pilot production of u-PA with porous microcarrier cell culture. Cytotechnology 33: 13-19, 2000.

19. Tanaka M, Obata T and Sasaki T: Evaluation of antitumor effects of docetaxel (Taxotere) on human gastric cancer in vitro and in vivo. Eur J Cancer 32A: 226-230, 1996.

20. Evans BD, Smith IE, Shorthouse AJ and Millar JL: A comparison of the response of human lung carcinoma xenografts to vindesine and vincristine. Br J Cancer 45: 466-468, 1982.

21. Wolff AC, Hammond ME, Schwartz JN, Hagerty KL, Allred DC, Cote RJ, Dowsett M, Fitzgibbons PL, Hanna WM, Langer A, et al: American Society of Clinical Oncology/College of American Pathologists guideline recommendations for human epidermal growth factor receptor 2 test in breast cancer. J Clin Oncol 25: 118-145, 2007.

22. Glasgow MD and Chougule MB: Recent developments in active tumor targeted multifunctional nanoparticles for combination chemotherapy in cancer treatment and imaging. J Biomed Nanotechnol 11: 1859-1898, 2015.

23. Griffiths R, Mikhael J, Gleeson M, Danese M and Dreyling M: Addition of rituximab to chemotherapy alone as first-line therapy improves overall survival in elderly patients with mantle cell lymphoma. Blood 118: 4808-4816, 2011.

24. Seal MD, Speers CH, O'Reilly S, Gelmon KA, Ellard SL and Chia SK: Outcomes of woman with early-stage breast cancer receiving adjuvant trastuzumab. Curr Oncol 19: 197-201, 2012.

25. Altomare I, Bendell JC, Bullock KE, Uronis HE, Morse MA, Hsu SD, Zafar SY, Blobe GC, Pang H, Honeycutt W, et al: A phase II trial of bevacizumab plus everolimus for patients with refractory metastatic colorectal cancer. Oncologist 16: 1131-1137, 2011.

26. Fleitas T, Martínez-Sales V, Vila V, Reganon E, Mesado D, Martín M, Gómez-Codina J, Montalar J and Reynés G: Circulating endothelial cells and microparticles as prognostic markers in advanced non-small cell lung cancer. PLoS One 7: e47365, 2012.

27. Vacchelli E, Pol J, Bloy N, Eggermont A, Cremer I, Fridman WH, Galon J, Marabelle A, Kohrt H, Zitvogel L, et al: Trial watch: Tumor-targeting monoclonal antibodies for oncological indications. Oncoimmunology 4: e985940, 2015.

28. Kargiotis O, Chetty C, Gogineni V, Gondi CS, Pulukuri SM, Kyritsis AP, Gujrati M, Klopfenstein JD, Dinh DH and Rao JS: uPA/uPAR downregulation inhibits radiation-induced migration, invasion and angiogenesis in IOMM-Lee menigioma cells and decreases tumors growth in vivo. Int J Oncol 33: 937-947, 2008.

29. Rabbani SA and Gladu J: Urokinase receptor antibody can reduce tumors volume and detect the presence of occult tumors metastases in vivo. Cancer Res 62: 2390-2397, 2002. 
30. Kobayashi H, Gotoh J, Shinohara H, Moniwa N and Terao T: Inhibition of the metastasis of Lewis lung carcinoma by antibody against urokinase-type plasminogen activator in the experimental and spontaneous metastasis model. Thromb Haemost 71: 474-480, 1994

31. Beloglazova IB, Beabealashvilli RSh, Gursky YG, Bocharov EV, Mineev KS, Parfenova EV and Tkachuk VA: Structural investigations of recombinant urokinase growth factor-like domain. Biochemistry (Mosc) 78: 517-530, 2013.

32. Bifulco K, Longanesi-Cattani I, Franco P, Pavone V, Mugione P Di Carluccio G, Masucci MT, Arra C, Pirozzi G, Stoppelli MP and Carriero MV: Single amino acid substitutions in the chemotactic sequence of urokinase receptor modulate cell migration and invasion. PLoS One 7: e44806, 2012.

33. Crowley CW, Cohen RL, Lucas BK, Liu G, Shuman MA and Levinson AD: Prevention of metastasis by inhibition of the urokinase receptor. Proc Natl Acad Sci USA 90: 5021-5025, 1993.

34. Indira Chandran V, Eppenberger-Castori S, Venkatesh T, Vine KL and Ranson M: HER2 and UPAR cooperativity contribute to metastatic phenotype of HER2-positive breast cance. Oncoscience 2: 207-224, 2015.

35. Oliveras-FerrarosC, Corominas-FajaB,CufíS, Vazquez-MartinA, Martin-Castillo B, Iglesias JM, López-Bonet E, Martin AG and Menendez JA: Epithelial-to-mesenchymal transition (EMT) confers primary resistance to trastuzumab (Herceptin). Cell Cycle 11: 4020-4032, 2012.

36. Capelan M, Pugliano L, De Azambuja E, Bozovic I, Saini KS, Sotiriou C, Loi S and Piccart-Gebhart MJ: Pertuzumab: New hope for patients with HER2-positive breast cancer. Ann Oncol 24: 273-282, 2013.

37. Gajria D and Chandarlapaty S: HER2-amplified breast cancer: Mechanisms of trastuzumab resistance and novel targeted therapies. Expert Rev Anticancer Ther 11: 263-275, 2011.
38. Baylin SB and Jones PA: A decade of exploring the cancer epigenome-biological and translational implications. Nat Rev Cancer 11: 726-734, 2011

39. Esteller M: Cancer epigenomics: DNA methylomes and histone-modification maps. Nat Rev Genet 8: 286-298, 2007.

40. Meng S, Tripathy D, Shete S, Ashfaq R, Saboorian H, Haley B, Frenkel E, Euhus D, Leitch M, Osborne C, et al: uPA and HER-2 gene status in individual breast cancer cells from blood and tissues. Proc Natl Acad Sci USA 103: 17361-17365, 2006.

41. Pierga JY, Bonneton C, Magdelénat H, Vincent-Salomon A, Nos C, Boudou E, Pouillart P, Thiery JP and de Cremoux P: Real-time quantitative PCR determination of urokinase-type plasminogen activator receptor (uPAR) expression of isolated micrometastatic cells from bone marrow of breast cancer patients. Int J Cancer 114: 291-298, 2005.

42. Liu D, Aguirre Ghiso J, Estrada Y and Ossowski L: EGFR is a transducer of the urokinase receptor initiated signal that is required for in vivo growth of a human carcinoma. Cancer Cell 1: 445-457, 2002

43. Jo M, Thomas KS, O'Donnell DM and Gonias SL: Epidermal grow th factor receptor-dependent and -independent cell-signaling pathways originating from the urokinase receptor. J Biol Chem 278: 1642-1646, 2003.

44. Guerrero J, Santibañez JF, González A and Martínez J: EGF receptor transactivation by urokinase receptor stimulus through a mechanism involving Src and matrix metalloproteinases. Exp Cell Res 292: 201-208, 2004.

45. Li C, Cao S, Liu Z, Ye X, Chen L and Meng S: RNAi-mediated downregulation of uPAR synergizes with targeting of HER2 through the ERK pathways in breast cancer cells. Int J Cancer 127: 1507-1516, 2010. 